\title{
Numerical simulations of solar macrospicules
}

\author{
K. Murawski ${ }^{1}$, A. K. Srivastava ${ }^{2}$, and T. V. Zaqarashvili ${ }^{3,4}$ \\ 1 Group of Astrophysics, UMCS, ul. Radziszewskiego 10, 20-031 Lublin, Poland \\ e-mail: kmur@kft.umcs. lublin.pl \\ 2 Aryabhatta Research Institute of Observational Sciences (ARIES), Manora Peak, Nainital-263 129, Uttarakhand, India \\ e-mail: aks@aries.res.in \\ 3 Space Research Institute, Austrian Academy of Sciences, Schmiedlstrasse 6, 8042 Graz, Austria \\ e-mail: teimuraz.zaqarashvili@oeaw.ac.at \\ 4 Abastumani Astrophysical Observatory at Ilia State University, University Str. 2, Tbilisi, Georgia \\ Received 28 June 2011 / Accepted 2 September 2011
}

\section{ABSTRACT}

\begin{abstract}
Context. We consider a localized pulse in the component of velocity, parallel to the ambient magnetic field lines, that is initially launched in the solar chromosphere.

Aims. We aim to generalize our recent numerical model of spicule formation by implementing a VAL-C model of solar temperature. Methods. With the use of the code FLASH we solve two-dimensional ideal magnetohydrodynamic equations numerically to simulate the solar macrospicules.

Results. Our numerical results reveal that the pulse located below the transition region triggers plasma perturbations, which exhibit many features of macrospicules. We also present an observational (SDO/AIA $304 \AA$ ) case study of the macrospicule that approximately mimics the numerical simulations.

Conclusions. In the frame of the model we devised, the solar macrospicules can be triggered by velocity pulses launched from the chromosphere.
\end{abstract}

Key words. magnetohydrodynamics (MHD) - instabilities - Sun: atmosphere

\section{Introduction}

Spicules are thin, cool, and dense structures that are observed in the solar limb (Beckers 1968, 1972; Suematsu 1998; Sterling 2000; Zaqarashvili \& Erdélyi 2009). Type I spicules are seen to be formed at an altitude of about $2000 \mathrm{~km}$ where they reveal a speed of $25 \mathrm{~km} \mathrm{~s}^{-1}$, reach a maximum level and then either disappear or fall off to the photosphere. A typical lifetime of spicules is 5-15 min with an average value of $\sim 7 \mathrm{~min}$ (Pasachoff et al. 2009). Spicules consist of double-thread structures (Tanaka 1974; Dara et al. 1998; Suematsu et al. 2008) and they reveal the bi-directional flow (Tsiropoula et al. 1994; Tziotziou et al. 2003, 2004; Pasachoff et al. 2009). Typical electron temperature and electron density in spicules are respectively $(15-17) \times 10^{3} \mathrm{~K}$ and $2 \times 10^{11}-3.5 \times 10^{10} \mathrm{~cm}^{-3}$ at altitudes of 4-10 Mm above the solar surface (Beckers 1968). As a result, spicules are much cooler and denser than ambient coronal plasma. High-resolution observations by Solar Optical Telescope onboard Hinode have revealed another type of spicule with many features different than classical limb spicules, and they are referred as type II spicules (De Pontieu et al. 2007). The mean diameter of type I spicules is estimated as $660 \pm 200 \mathrm{~km}$ (Pasachoff et al. 2009), but the type II spicules have smaller diameters $(\leq 200 \mathrm{~km})$ in the Ca II H line (De Pontieu et al. 2007). While observed in the $\mathrm{H}_{\alpha}$ line, type I spicules can reach up to 4-12 Mm in height from the solar limb with a mean value of $7200 \pm 2000 \mathrm{~km}$ (Pasachoff et al. 2009). The type II spicules are significantly shorter. The main differences between type I and type II spicules are (a) the lifetime, the former lasts longer; (b) the evolution, the former usually shows a parabolic profile in $\mathrm{Ca}$ II H, while the latter shows an upflow and then disappears; (c) the former usually reveal lower velocities (e.g., De Pontieu et al. 2007, 2009; Rouppe Van der Voort et al. 2009).

Additionally, very long spicules, called macrospicules with typical lengths of up to $40 \mathrm{Mm}$ and with higher temperatures are frequently observed mostly near the polar regions (Cook et al. 1984; Pike \& Harrison 1997; Ashbourn \& Woods 2005). Georgakilas et al. (1999) have observed the giant solar spicules that reach at a maximum height of about $12-15 \mathrm{Mm}$ with their lifetime ranging between five and twelve minutes, with an ejection velocity around $\sim 50 \mathrm{~km} \mathrm{~s}^{-1}$. Wilhelm (2000) has also observed the largest macrospicules upto a height of $40 \mathrm{Mm}$ in the north polar coronal hole. In conclusion, the solar macrospicules can extend from $7 \mathrm{Mm}$ to $45 \mathrm{Mm}$ above the solar limb with a lifetime of 3-45 min, and they are mostly concentrated in the polar coronal holes (Sterling 2000).

In spite of various theoretical models to explain the spicule ejection in the lower solar atmosphere, many recent numerical methods have been developed to simulate the solar spicules/macrospicules with an energy input at their base in the photosphere as a pressure pulse or an Alfvén wave that steepens into a shock wave (Sterling 2000, and references therein). Recently, Hansteen et al. (2006) and De Pontieu et al. (2007) have simulated the formation of dynamic fibrils due to slow magneto-acoustic shocks through two-dimensional (2D) numerical simulations. They suggest that these shocks are formed when acoustic waves generated by convective flows and global p-modes in the lower lying photosphere leak upward into the magnetized chromosphere. Heggland et al. (2007) used the initial periodic piston to drive the upward propagating shocks in 1D simulation and Martinez-Sykora et al. (2009) considered the emergence of new magnetic flux, but the drivers of 
spicules come from collapsing granules, energy release in the photosphere or lower chromosphere. However, these simulations could not mimic the double structures and bi-directional flows in spicules. On the other hand, Murawski \& Zaqarashvili (2010) performed 2D numerical simulations of magnetohydrodynamic (MHD) equations and showed that the 2D rebound shock model (Hollweg 1982) may explain both the double structures and bidirectional flows. They used a single initial velocity pulse, which leads to the formation of consecutive shocks due to nonlinear wake in the stratified atmosphere. However, they considered a simple model of atmospheric temperature that was approximated by a smoothed step function profile.

In this paper, we adopt a more appropriate temperature profile by Vernazza et al. (1981) that implements characteristic (Alfvén and tube) speed profiles to launch the pulse at the chromosphere. Our more general model reveals that macrospicules are effectively excited by velocity pulses launched at the chromosphere. Additionally, our model confirms the findings of Murawski \& Zaqarashvili (2010) who explained the observed properties of spicules (e.g., width, height, and bi-directional flows). We also present an observational case study using SDO/AIA $304 \AA$ of a macrospicule in the north polar region, which approximately matches the simulated macrospicule in the oblique magnetic field. This observed spicule appears near the north-east boundary of a moderately evolved hole at the polar cap, and moves off the limb obliquely.

This paper is organized as follows. A numerical model is presented in Sect. 2, and the corresponding numerical results are shown in Sect. 3. The observational case study of a macrospicule is presented in Sect. 4 in support of the numerical simulations. This paper is completed with a discussion and conclusions in Sect. 5.

\section{A numerical model}

We consider a gravitationally-stratified solar atmosphere that is described by the ideal 2D MHD equations:

$$
\begin{array}{r}
\frac{\partial \varrho}{\partial t}+\nabla \cdot(\varrho \boldsymbol{V})=0, \\
\varrho \frac{\partial \boldsymbol{V}}{\partial t}+\varrho(\boldsymbol{V} \cdot \nabla) \boldsymbol{V}=-\nabla p+\frac{1}{\mu}(\nabla \times \boldsymbol{B}) \times \boldsymbol{B}+\varrho \boldsymbol{g}, \\
\frac{\partial p}{\partial t}+\nabla \cdot(p \boldsymbol{V})=(1-\gamma) p \nabla \cdot \boldsymbol{V}, \quad p=\frac{k_{\mathrm{B}}}{m} \varrho T, \\
\frac{\partial \boldsymbol{B}}{\partial t}=\nabla \times(\boldsymbol{V} \times \boldsymbol{B}), \quad \nabla \cdot \boldsymbol{B}=0 .
\end{array}
$$

Here $\varrho$ is mass density, $\boldsymbol{V}=\left[V_{x}, V_{y}, 0\right]$ the flow velocity, $\boldsymbol{B}=$ $\left[B_{x}, B_{y}, 0\right]$ the magnetic field, $p$ is gas pressure, $T$ temperature, $\gamma=5 / 3$ the adiabatic index, $\boldsymbol{g}=(0,-g, 0)$ gravitational acceleration of its value $g=274 \mathrm{~m} \mathrm{~s}^{-2}, m$ is mean particle mass, and $k_{\mathrm{B}}$ is the Boltzmann's constant.

\subsection{Equilibrium configuration}

We assume that the solar atmosphere is in static equilibrium $\left(\boldsymbol{V}_{\mathrm{e}}=\mathbf{0}\right)$ with a force-free magnetic field,

$\left(\nabla \times \boldsymbol{B}_{\mathrm{e}}\right) \times \boldsymbol{B}_{\mathrm{e}}=\mathbf{0}$.

As a result, the pressure gradient is balanced by the gravity force,

$-\nabla p_{\mathrm{e}}+\varrho_{\mathrm{e}} \boldsymbol{g}=\mathbf{0}$

Here the subscript ${ }_{\mathrm{e}}$ corresponds to equilibrium quantities.
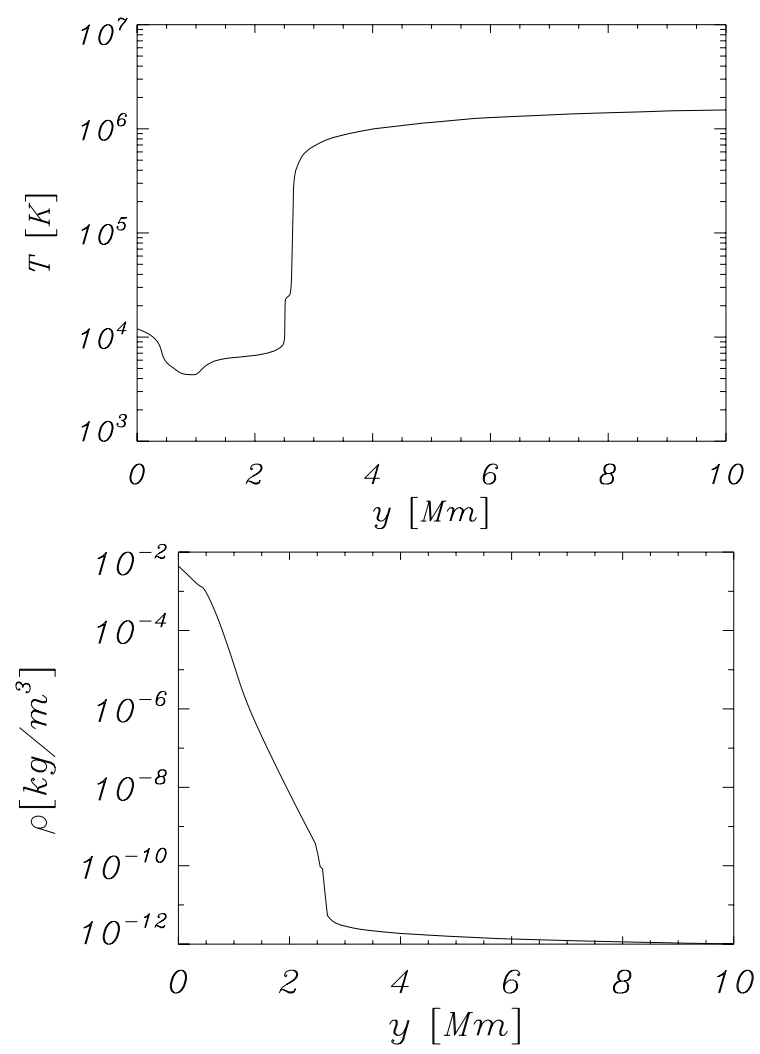

Fig. 1. Equilibrium profile of solar temperature (top panel) and mass density (bottom panel).

Using the ideal gas law and the $y$-component of hydrostatic pressure balance indicated by Eq. (6), we express equilibrium gas pressure and mass density as

$p_{\mathrm{e}}(y)=p_{0} \exp \left[-\int_{y_{\mathrm{r}}}^{y} \frac{\mathrm{d} y^{\prime}}{\Lambda\left(y^{\prime}\right)}\right], \quad \varrho_{\mathrm{e}}(y)=\frac{p_{\mathrm{e}}(y)}{g \Lambda(y)}$.

Here

$\Lambda(y)=k_{\mathrm{B}} T_{\mathrm{e}}(y) /(m g)$

is the pressure scale height, and $p_{0}$ denotes the gas pressure at the reference level that we choose in the solar corona at $y_{\mathrm{r}}=10 \mathrm{Mm}$.

We adopt an equilibrium temperature profile $T_{\mathrm{e}}(z)$ for the solar atmosphere that is close to the VAL-C atmospheric model of Vernazza et al. (1981). It is smoothly extended into the corona (Fig. 1, top). Then with the use of Eq. (7) we obtain the corresponding gas pressure and mass density profiles.

We assume that the initial magnetic field satisfies a currentfree condition, $\nabla \times \boldsymbol{B}_{\mathrm{e}}=\mathbf{0}$, and it is specified by the magnetic flux function, $A$, such that

$\boldsymbol{B}_{\mathrm{e}}(x, y)=\left[\frac{\partial A}{\partial y},-\frac{\partial A}{\partial x}, 0\right]$

with

$A(x, y)=S_{1} \frac{x-x_{1}}{\left(x-x_{1}\right)^{2}+\left(y-y_{1}\right)^{2}}$.

Here $S_{1}$ denotes a strength of magnetic moment that is located at $\left(x_{1}, y_{1}\right)$. We choose and hold fixed $x_{1}=0 \mathrm{Mm}$, $y_{1}=-40 \mathrm{Mm}$, and $S_{1}$ is specified from the requirement that at the reference point $\left(x_{\mathrm{r}}=0, y_{\mathrm{r}}=10\right)$ Mm Alfvén speed, 


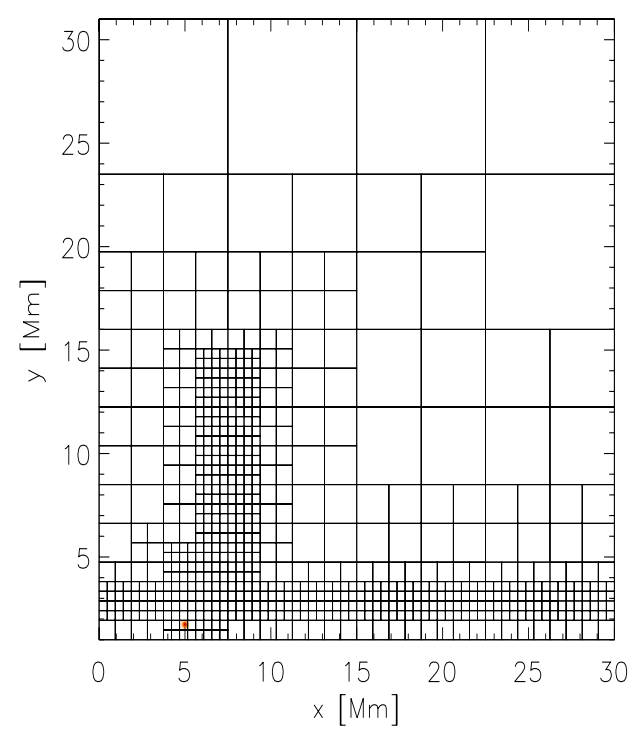

Fig. 2. The static grid system with block boundaries represented by solid lines for the case of $x_{0}=5 \mathrm{Mm}$. The initial perturbation of the system is displayed by the red dot at $(5.00,1.75) \mathrm{Mm}$.

$c_{\mathrm{A}}\left(x_{\mathrm{r}}, y_{\mathrm{r}}\right)=\left|\mathbf{B}_{\mathrm{e}}\left(x_{\mathrm{r}}, y_{\mathrm{r}}\right)\right| / \sqrt{\mu \varrho_{\mathrm{e}}\left(y_{\mathrm{r}}\right)}$, is ten times higher than the sound speed, $c_{\mathrm{s}}\left(y_{\mathrm{r}}\right)=\sqrt{\gamma p_{\mathrm{e}}\left(y_{\mathrm{r}}\right) / \varrho_{\mathrm{e}}\left(y_{\mathrm{r}}\right)}$.

For these settings magnetic field is predominantly vertical around $x=0 \mathrm{Mm}$, while around $(x=5, y=1.75) \mathrm{Mm}$ it is oblique with about $\pi / 3$ angle to the solar surface.

\subsection{Perturbations}

We initially perturb the above equilibrium impulsively by a Gaussian pulse in the component of velocity that is nearly parallel to ambient magnetic field lines, $V_{\|}$, viz.,

$V_{\|}(x, y, t=0)=A_{\mathrm{v}} \exp \left[-\frac{\left(x-x_{0}\right)^{2}+\left(y-y_{0}\right)^{2}}{w^{2}}\right]$.

Here $A_{\mathrm{v}}$ is the amplitude of the pulse, $\left(x_{0}, y_{0}\right)$ its initial position, and $w$ denotes its width. We set and hold fixed $A_{\mathrm{v}}=40 \mathrm{~km} \mathrm{~s}^{-1}$, $w=150 \mathrm{~km}$, and $y_{0}=1.75 \mathrm{Mm}$, while allowing $x_{0}$ to vary. We consider two cases: (a) $x_{0}=0 \mathrm{Mm}$ and (b) $x_{0}=5 \mathrm{Mm}$.

\section{Results of numerical simulations}

Equations (1)-(4) are solved numerically using the code FLASH (Lee \& Deane 2009). This code implements a second-order unsplit Godunov solver (e.g., Murawski 2002) with various slope limiters and Riemann solvers, as well as adaptive mesh refinement (AMR). We use the minmod slope limiter and the Roe Riemann solver (e.g., Toro 2009). For the case of (a) and (b) we set the simulation box as $(5,5) \mathrm{Mm} \times(1,41) \mathrm{Mm}$ and $(0,30) \mathrm{Mm} \times(1,31) \mathrm{Mm}$, respectively. We impose boundary conditions by fixing in time all plasma quantities at all four boundaries to their equilibrium values. In all our studies we use a static but non-uniform grid with a minimum (maximum) level of refinement set to 1 (7). See Fig. 2 for the grid system for the case of $x_{0}=5 \mathrm{Mm}$. A similar grid system was chosen for the case of $x_{0}=0 \mathrm{Mm}$. As the grid was static, no refinement strategy was adopted. As each block consists of $8 \times 8$ identical numerical cells, we reach the effective spatial resolution of about $60 \mathrm{~km}$.

We discuss two cases: (a) essentially vertical magnetic field and (b) oblique magnetic field. For case (a) we launch the initial pulse at $x_{0}=0 \mathrm{Mm}$, while case (b) is realized for $x_{0}=5 \mathrm{Mm}$. In the latter case the magnetic field creates the angle of about $\pi / 3$ to the horizontal direction.

\subsection{Essentially vertical magnetic field}

The temporal snapshots of the simulated macrospicule in the model atmosphere of the VAL-C temperature and essentially vertical magnetic field are shown in Fig. 3. It displays the spatial profiles of plasma temperature (colour maps) and velocity (arrows), resulting from the initial velocity pulse that was launched at $x_{0}=0 \mathrm{Mm}$. The upper left panel corresponds to $t=100 \mathrm{~s}$. Cold chromospheric plasma lags behind the shock front, which at this time reaches an altitude of $y \simeq 8 \mathrm{Mm}$. The reason for the material being lifted is the rarefaction of the plasma behind the shock front, which leads to low pressure there (Hansteen et al. 2006). As a result, the pressure gradient works against gravity and forces the chromospheric material to penetrate the solar corona.

It is noteworthy that small-amplitude vertically propagating waves are described by the Klein-Gordon equation (Lamb 1909). It follows from this equation that initial, spatially-localized perturbation results in a wavefront that moves away from the launching place. The disturbance ahead of the wavefront is at rest, but behind the wavefront an oscillation wake results in. This wake oscillates with essentially constant frequency, and the amplitude of the oscillations behind the wavefront declines gradually in time. As a consequence of the equilibrium mass density falls off with height, the amplitude of these oscillations grows with altitude and then the linear theory is not valid anymore because nonlinear effects result in trailing shocks with the secondary shock following the leading shock (Hollweg 1982).

The next snapshot (top middle panel) is drawn for $t=200 \mathrm{~s}$, when the macrospicule rises to the altitude of $y \simeq 11 \mathrm{Mm}$. At $t=300 \mathrm{~s}$ (top-right panel) the macrospicule has already subsided. Because the secondary shock lifts up the chromospheric material (see two small peaks on both sides of the macrospicule), there are upward flows at the macrospicule sides, well seen at $t=400 \mathrm{~s}$ (middle-left panel). It is clear from the snapshots at $t \geq 200 \mathrm{~s}$ that the plasma exhibits some horizontal oscillations. This is because the plasma pushes the magnetic field lines outside owing to the high value of plasma $\beta$ at the chromosphere. The signatures of these horizontal oscillations that are running along the transition region-corona interface. There are well seen bi-directional flows in the macrospicule: downward at the centre and upward at the boundaries, which agrees with the observational data (Tsiropoula et al. 1994; Tziotziou et al. 2003, 2004; Pasachoff et al. 2009). Eventually, for the downward streaming plasma follows a path in the cool core region as seen at $t=800 \mathrm{~s}$. Since the macrospicule plasma at its leading edge enters in the corona, it may be heated quickly at the coronal temperature, and associated with the greater upflow velocity typically in the range of $20-40 \mathrm{~km} \mathrm{~s}^{-1}$ compared to the background in case of both singular and bi-directional flows (cf., Fig. 3). Therefore, this segment of macrospicule may appear in the form of a Doppler blue shift of the centroid of FUV/EUV lines formed with maximum ionic fraction at their typical coronal/TR formation temperatures. The blue shift and plasma upflows with the typical macrospicule rise-up speed have been observed by Wilhelm (2000) and Suematsu et al. (1995). However, the scenario of temperature, flow, and magnetic field structures in the bottom part of the macrospicules is rather complex. The cool core, steep temperature gradient towards boundary may evolve at the base. The up and down-flow structuring (cf. Fig. 3) may be 

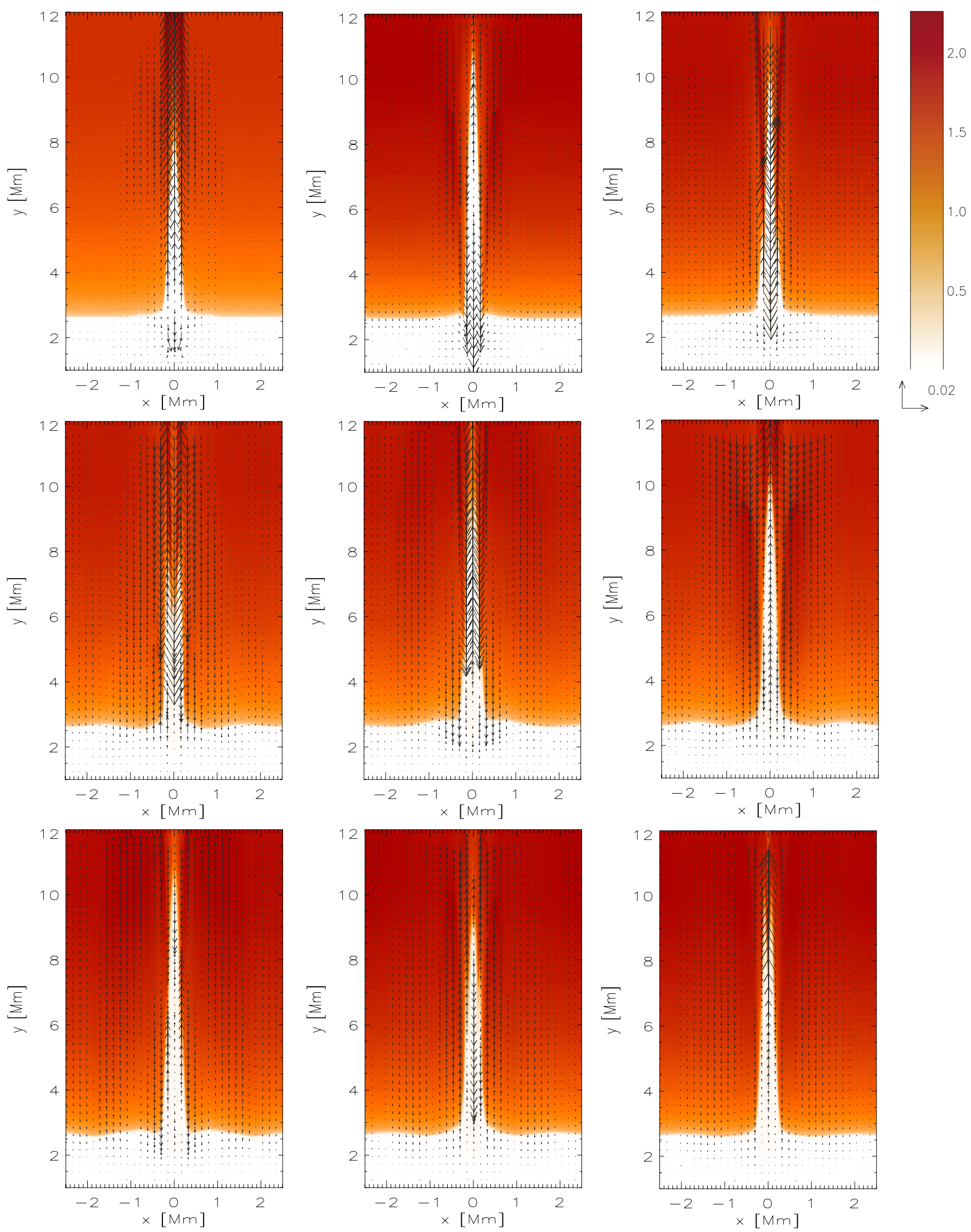

Fig. 3. Temporal snapshots of simulated macrospicule in the case of essentially vertical magnetic field (see Sect. 3.1). The temperature (colour maps) and velocity (arrows) profiles at $t=100 \mathrm{~s}, t=200 \mathrm{~s}, t=300 \mathrm{~s}, t=400 \mathrm{~s}, t=500 \mathrm{~s}, t=600 \mathrm{~s}, t=700 \mathrm{~s}, t=800 \mathrm{~s}$, and $t=10^{3} \mathrm{~s}$ (from top to bottom) for $x_{0}=0 \mathrm{Mm}$. Temperature is drawn in units of $1 \mathrm{MK}$. The arrow below each panel represents the length of the velocity vector, expressed in units of $30 \mathrm{~km} \mathrm{~s}^{-1}$. The colour bar is common to all the panels. 


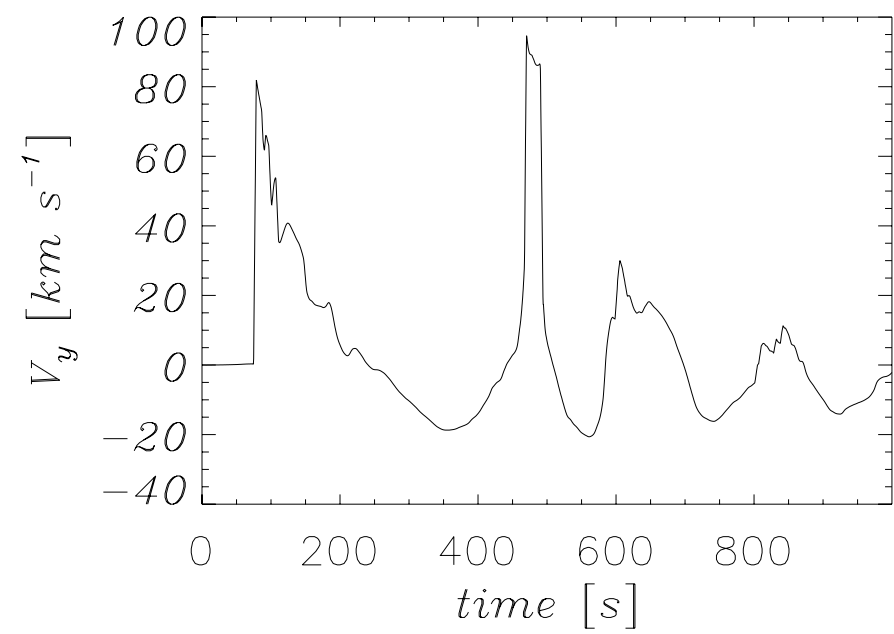

Fig. 4. Time signature of velocity $V_{y}$ collected at $(x=0, y=14) \mathrm{Mm}$ for the case of an essentially vertical magnetic field as shown in Fig. 3.

observed in form of a mixed red and blue-shift scenario as previously explained by Wilhelm (2000) in the pass band of cool chromospheric lines.

The middle-right panel is drawn for $t=600 \mathrm{~s}$, which clearly resembles a macrospicule-like structure with the chromospheric temperature and mass density. Its width and mean rising speed are about $0.4 \mathrm{Mm}$ and $30 \mathrm{~km} \mathrm{~s}^{-1}$, respectively. These values are close to the corresponding characteristics of typical macrospicules. However, this is just a presentation of the simulation case study of the solar macrospicule in an essentially vertical magnetic field configuration. The typical length, width, speed, and life time can vary by tuning the initial conditions of the numerical model. Afterwards the material again falls back at the central part, while the next shock forces the plasma to move upwards at the spicule boundaries. We see quasi-recurrently single $(t=200 \mathrm{~s}, t=600 \mathrm{~s}, t=800 \mathrm{~s})$, double (e.g., $t=400 \mathrm{~s})$, and triple (e.g. $t=300 \mathrm{~s}$ ) structures, which are characteristic features of macrospicules.

Figure 4 illustrates the vertical component of velocity that is collected in time at the detection point $(x=0, y=14) \mathrm{Mm}$ for the case of Fig. 3. As a result of fast mass density fall off with height, upwardly propagating waves grow in their amplitude and steepen rapidly into shocks. The arrival of the first shock front at the detection point, $y=14 \mathrm{Mm}$, is clearly seen at $t \simeq 70 \mathrm{~s}$. The second shock front reaches the detection point at $t \simeq 460 \mathrm{~s}$, i.e., after $\sim 290 \mathrm{~s}$. This secondary shock results from the non-linear wake, which lags behind the leading shock.

\subsection{Oblique magnetic field}

The temporal snapshots of the simulated macrospicule in the model atmosphere of the VAL-C temperature and oblique magnetic field are shown in Fig. 5. It illustrates the spatial profiles of plasma temperature (colour maps) and vertical velocity (arrows) resulting from the initial velocity pulse that was launched at $x_{0}=5 \mathrm{Mm}$. As a result, the plasma flows along the inclined magnetic field. The dynamics of plasma essentially resembles what is obtained by vertically propagating pulse. At $t=200 \mathrm{~s}$ the velocity signal reaches the altitude of up to $8 \mathrm{Mm}$. Then it falls back again resembling the consecutive double structure and bi-directional flows.

Figure 6 illustrates the vertical component of velocity that is collected in time at the detection point $\left(x_{\mathrm{d}}=8.5, y_{\mathrm{d}}=14\right) \mathrm{Mm}$ for the case of Fig. 5. The arrival of the first shock front at the detection point is clearly seen at $t \simeq 100 \mathrm{~s}$. The second shock arrives at the detection point at $t=430 \mathrm{~s}$ i.e., after $\sim 330 \mathrm{~s}$. Another two shocks appear at later times, that is, at $\sim 650 \mathrm{~s}$ and $\sim 900 \mathrm{~s}$.

However, this is also a presentation of the simulation case study of the solar macrospicule in oblique magnetic field configuration driven by a velocity pulse in the model temperature of the solar atmosphere. The typical length, width, speed, and life time of the obliquely propagating macrospicules can also vary by tuning the initial conditions of the numerical model.

\section{Observational evidence of a pulse-driven macrospicule}

In the present section, we describe an observational signature of a limb spicule, which approximately resembles the simulated macrospicule as driven by a velocity pulse in the more general oblique magnetic field configuration. We use time-series data of a solar spicule at the north polar region as observed in $304 \AA$ filter of Atmospheric Assembly Imager (AIA) onboard the Solar Dynamics Observatory (SDO) on 3 August 2010 during 03:12:50 UT-03:22:50 UT. The SDO/AIA has a typical resolution of 0.6 " per pixel and the highest cadence of $12 \mathrm{~s}$, and it observed the full solar disk in three UV $(1600 \AA, 1700 \AA$, $4500 \AA$ ) and seven EUV (171 ̊, $193 \AA$ A, $211 \AA, 94 \AA, 304 \AA$, $335 \AA, 131 \AA$ ) wavelengths. Therefore, it provides the unique observations of multi-temperature, high-resolution, and hightemporal plasma dynamics all over the Sun. The field-of-view of the north polar region as observed by SDO/AIA $304 \AA$ on 03 August 2010 was $\left(1094^{\prime \prime}, 317^{\prime \prime}\right)$, while the $\left(X_{\text {cen }}, Y_{\text {cen }}\right)$ was $\left(-29.618^{\prime \prime}, 918.631^{\prime \prime}\right)$. The time series was obtained by the SSW cutout service at LMSAL, USA, which was corrected for the flatfield and spikes. We ran aia_prep subroutine of SSW IDL also for further calibration and cleaning of the time series data.

We observed a giant spicule dynamics at the limb of north polar region. The macrospicule was launched obliquely from the background open field lines of the polar coronal hole. The life time of the spicule was observed as $\sim 10 \mathrm{~min}$, which fits with the typical life time of the spicules/macrospicules (Georgakilas et al. 1999; Sterling 2000; Pasachoff et al. 2009). The observed macrospicule reaches upto a height of $\sim 12 \mathrm{Mm}$ and has the width of $\sim 2 \mathrm{Mm}$. It should be noted that the present observation is only a case study in the support of the numerical simulation of a solar macrospicule. Macrospicules may reach of 7-40 Mm heights with a typical life time of 3-45 min (Sterling 2000). Our aim here is only to show that these observations reveal to some extent the nature of the spicule according to the numerical modeling.

The spicule starts moving up above the solar limb on $\sim 03: 13$ UT and reaches at a maximum height of $\sim 12 \mathrm{Mm}$ at $\sim 03: 17$ UT with approximate average rising speed of $\sim 48 \mathrm{~km} \mathrm{~s}^{-1}$. The height of $\sim 12 \mathrm{Mm}$, life time of $\sim 10 \mathrm{~min}$, and the speed of $\sim 48 \mathrm{~km} \mathrm{~s}^{-1}$ are typical values of the observed macrospicules (Georgakilas et al. 1999; Sterling 2000). The unique evidence this macrospicule presented is how it rises obliquely off the limb during $\sim 250 \mathrm{~s}$ of its life time, and then falls back along the same path. The simulated macrospicule in the oblique magnetic field configuration (cf., Fig. 5) also reaches a height of $\sim 8 \mathrm{Mm}$ in $\sim 200 \mathrm{~s}$, and then its material attempts to fall back. The falling material of the simulated macrospicule is encountered by the wave train of the another upcoming pulses that uplift the spicule material quasi-periodically up to a certain height again and again in the solar atmosphere. The observed dynamics of macrospicule also supports its being formed by a 
A\&A 535, A58 (2011)
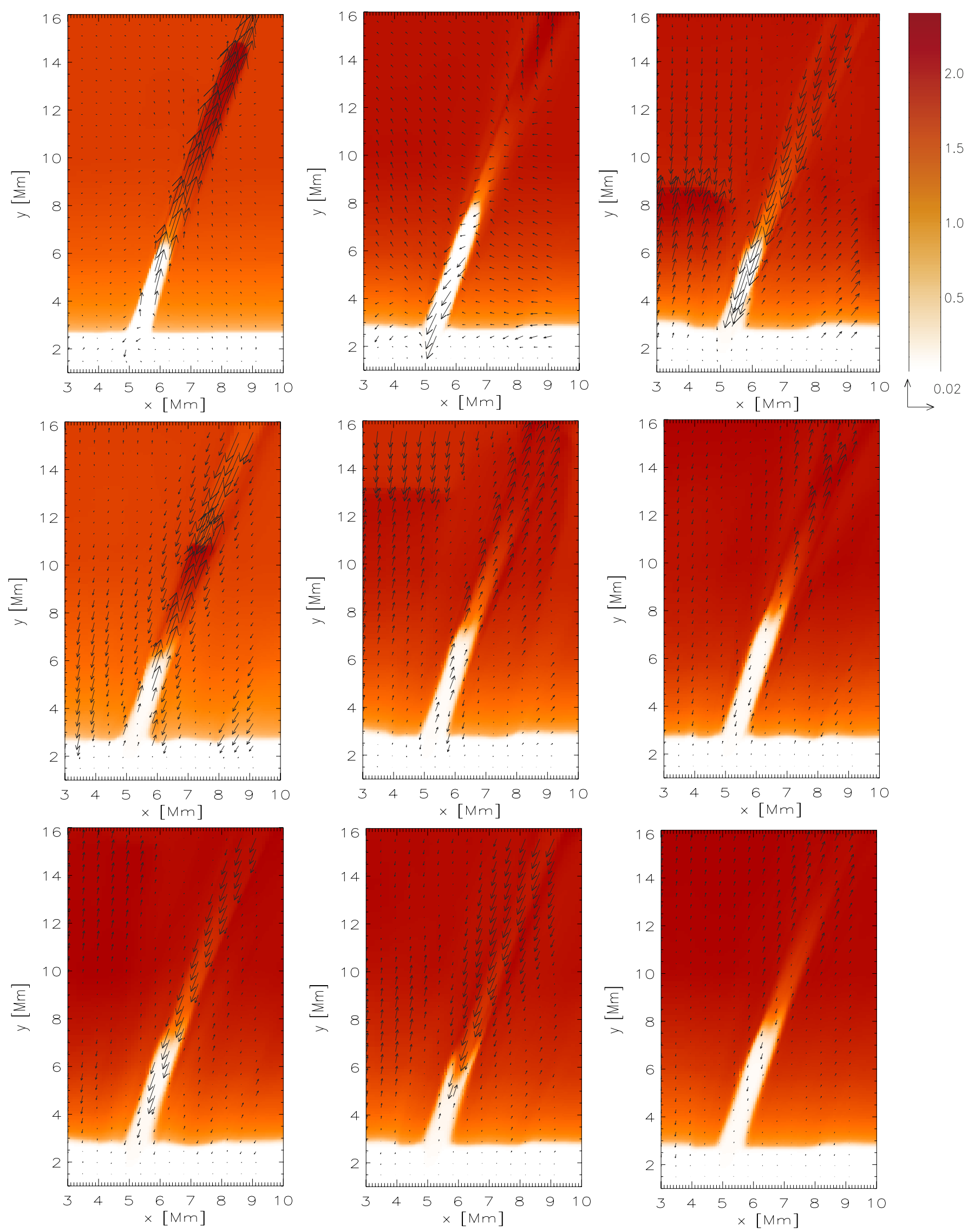

Fig. 5. Same as Fig. 3, but for the case of an oblique magnetic field (see Sect. 3.2). 


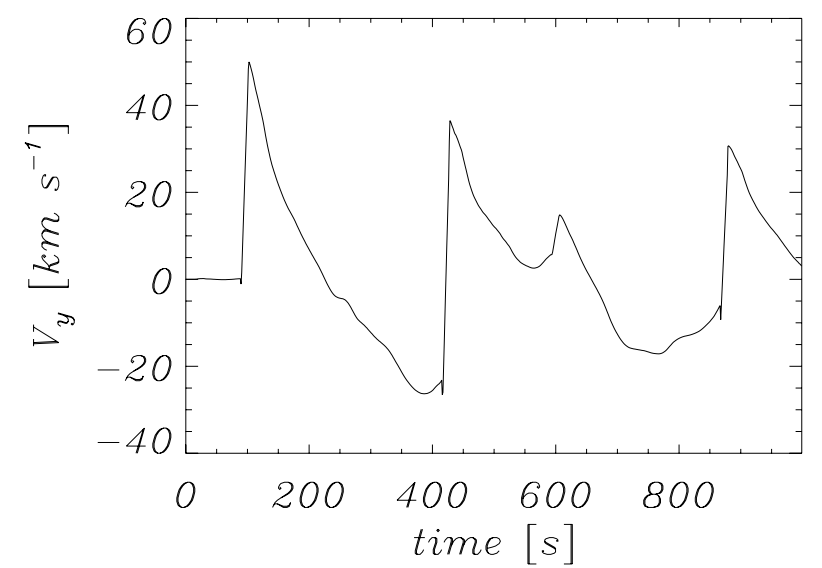

Fig. 6. Same as Fig. 4, but for the case of an oblique magnetic field shown in Fig. 5.

similar velocity pulse that triggered in the chromosphere and steepens in the form of a shock in the transition region/corona. It should be noted that the rising time of the observed macrospicule is $\sim 250 \mathrm{~s}$; however, its falling time is longer around $\sim 350 \mathrm{~s}$. This means that the falling cool plasma may interact with the uplifted plasma coming from the lower solar atmosphere along the same path of the spicule. It is also clear from the snapshots during 03:16 UT-03:23 UT that the falling plasma is trying to settle down, while some brightened material is being pushed up from the lower part of atmosphere. However, the signal in the line $304 \AA$ filter is not necessarily optically thick, and other contributions from the line-of-sight signal may contaminate the observations. Therefore, it is not completely clear that the brightening at the footprint is due to the rebound compression. After reaching in the upper atmosphere along the magnetic field lines, this spicule falls back, and cool plasma traced back its backward path as observed here.

Despite these uncertainties, the observed feature is approximately similar to the simulated macrospicule in the oblique magnetic field. In the case of the observed spicule, the plasma finally settles down. The reason may be that the upcoming pulses in the real atmosphere may not have the same amplitude and spatio-temporal distribution at the base of the spicule. The decay of the wave train of the pulses probably does not allow the quasi-periodic rise and fall of the spicule plasma from the same place. The downflowing material may also suppress the upcoming pulses that may finally allow the settling down of the spicule plasma.

The observed spicule is two times wider than the simulated one. However, the mean rising time and maximal height are almost closer for observed and simulated macrospicules. The height of the simulated spicule can be tuned exactly to the observations by a small adjustment of the velocity pulse. Simulated and observed velocities also match the typical velocities of the macrospicules. The fine tuning of the initial conditions in the numerical simulation can mimic the observed conditions exactly; however, here our aim is only to present an observational case study of the macrospicule to reveal its pulse-driven nature. The resolution and real complex conditions of the solar atmosphere do not permit us to precisely study the quasi-periodic rise and fall of the spicule plasma at the same place in the observations as were evident in the simulations. However, life time of the falling plasma and some observational evidence in the time series (03:17-03:23 UT) indicate interaction of falling material with the element of rising plasma. This may be due to the arrival of another pulse from below the chromosphere, which may try to push the plasma opposing the gravitational free fall.

In conclusion, the observations of the macrospicule resembles the simulation of pulse driven macrospicules. One more interesting point is noticeable in the observations that the spicule is being generated near the boundary of a brightened network at the north polar coronal hole. This network may be the place of the launching of a velocity pulse that triggers the macrospicule higher in the solar atmosphere. It should be noted that all the spicules/macrospicules do not show similar properties to those we observe here. Therefore, the activity in the associated brightened magnetic network may be the most probable cause for the initial velocity pulse to trigger the observed macrospicule. However, this is just a case study to support the numerical simulations presented in the paper, and the measured spatial and temporal scales are approximate when compared to the simulations. The detailed observational search of the solar macrospicules that match the numerical simulations exactly, will be done in our future projects.

\section{Discussion and conclusions}

The formation of solar macrospicules is still an unresolved problem in solar physics. Several competitive mechanisms have been supposed from time to time, but none of them could explain all the properties of macrospicules. We performed 2D numerical simulation of the velocity pulse, which was launched at the chromosphere, in stratified solar atmosphere with the VAL-C temperature profile. The amplitude of upward propagating perturbation rapidly grows with height due to the rapid decrease in the equilibrium mass density. Therefore, the perturbation quickly steepens into the shock in upper regions of the solar chromosphere that launches the cool spicule material behind it (Hansteen et al. 2006). The simulated properties of the macrospicules both in the vertical and oblique magnetic field configurations, e.g., velocity, height, life-time, bi-directional flows etc., clearly match with the typical properties of this type of giant spicules.

We also present a case study of the observed macrospicule using the recent observations from SDO/AIA in $304 \AA$ to support the observations. The observed macrospicule rises obliquely off the limb in the north polar region and reaches a height of $\sim 12 \mathrm{Mm}$ in the first four minutes of its total life time of $\sim 10 \mathrm{~min}$. These observed phenomena and parameters support the simulated macrospicule in the oblique magnetic field configuration. The width of the observed spicule is, however, comparatively larger than the simulated one. But, this is one of the free parameters of the numerical simulation that can be tuned with the shape and size of the velocity pulse to trigger such macrospicules. The amplitude of the velocity pulse can also tune the rising speed of the simulated spicule. The observed spicule rises up and then falls back along the same path just like the simulated pulse driven macrospicule. The doubly split head of the observed spicule is also evident in the observations once it approaches the maximum height. This may indicate the initiation of bi-directional plasma flows along the spicule as similar to the numerical simulations. However, the baseline of the observations does not allow us to examine the interaction of the falling spicule material with the uplifting plasma. However, the longer falling phase compared to the rising one may indicate some interaction between falling and rising plasmas. Unfortunately, we do not have the Doppler velocity fields and related observations to comment more on this aspect. However, we can easily identify some upward motion of dense bright plasma near the spicule base around 03:16-03:21 UT that may oppose the falling plasma 

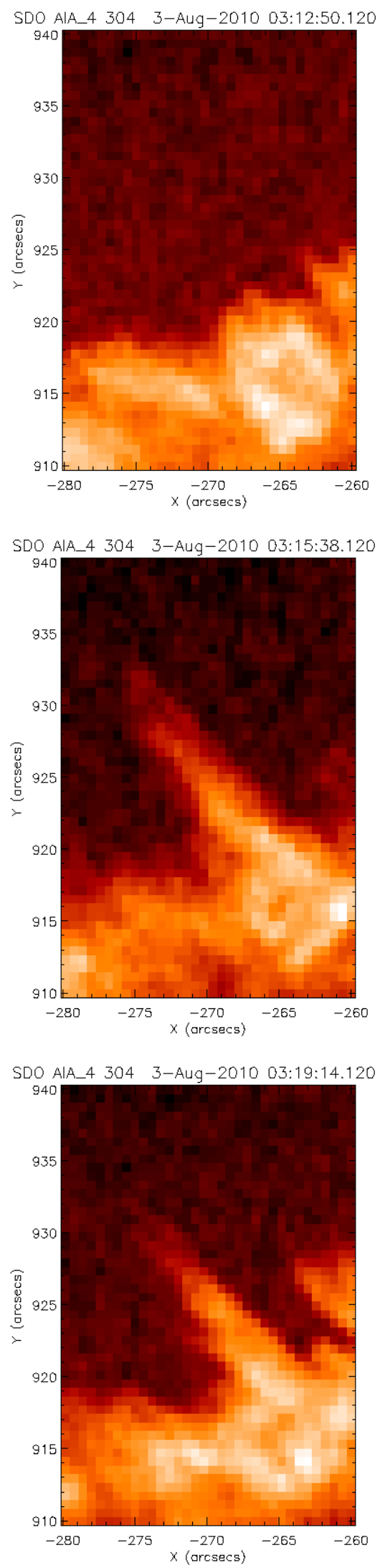
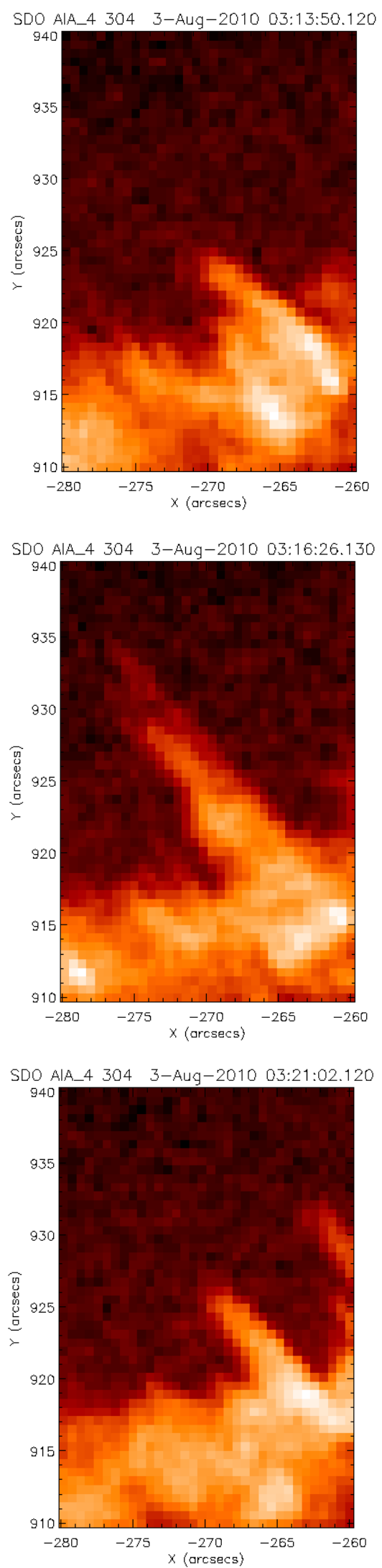
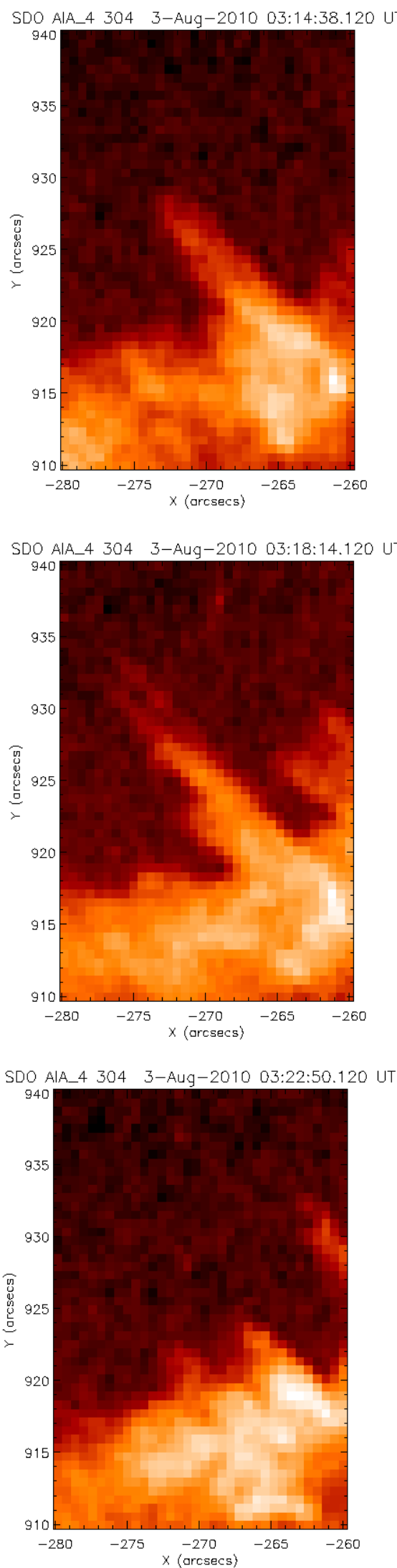

Fig. 7. The SDO/AIA $304 \AA$ A time sequence of a macrospicule propagation above the solar limb in the north polar region, clearly shows the upward motion of the plasma and its downward motion along the same path. 
that is already being lifted up to a maximum height of $\sim 12 \mathrm{Mm}$. When such an interaction occurs, the falling plasma probably subsiding and shows the two heads as a double component as also reported in the simulation snapshot of $400 \mathrm{~s}$ in Fig. 5.

In conclusion, our numerical simulations of the solar macrospicules exhibit approximately observed properties. We should admit that the performed 2D simulations are ideal in the sense that they do not include radiative transfer and thermal conduction along field lines. The field configuration and the initial stratification are simple and in hydrostatic equilibrium. Since we did not attempt to synthesize observables, the exact comparison with observations is unlikely. The detailed observational search of the solar macrospicules matching the numerical simulations exactly, will be done in our future projects. Simultaneous imaging and spectroscopic observations should also be performed in future to extensively explore the dynamics of such macrospicules that will also impose rigid constraints on the numerical simulation models.

Acknowledgements. The authors express their thanks to the referee for his/her comments. K.M. thanks Kamil Murawski for his assistance in drawing numerical data. A.K.S. acknowledges Shobhna Srivastava for the patient encouragements. The work of T.Z. was supported by the Austrian Fonds zur Förderung der wissenschaftlichen Forschung (project P21197-N16) and the Georgian National Science Foundation (under grant GNSF/ST09/4-310). The software used in this work was in part developed by the DOE-supported ASC/Alliance Center for Astrophysical Thermonuclear Flashes at the University of Chicago. We acknowledge the use of the SDO/AIA observations for this study. The data is provided courtesy of NASA/SDO, LMSAL, and the AIA, EVE, and HMI science teams.

\section{References}

Ashbourn, J. M. A., \& Woods, L. C. 2005, Physica Scripta, 71, 123 Beckers, J. M. 1968, Sol. Phys., 3, 367

Beckers, J. M. 1972, ARA\&A, 10, 73
Cook, J. W., Breuckner, G. E., Bartoe, J.-D. F., \& Socker, D. G. 1984, AdSpR, 4,59

Dara, H. C., Koutchmy, S., \& Suematsu, Y. 1998, Solar Jets and Coronal Plumes, ESA SP, 421, 255

De Pontieu, B., McIntosh, S., Hansteen, V. H., et al., 2007, PASJ, 59, S655

De Pontieu, B., McIntosh, S. W., Hansteen, V. H., \& Schrijver, C. J. 2009, ApJ, 701, L1

De Pontieu, B., McIntosh, S. W., Carlsson, M., et al. 2011, Science, 331, 55

Georgakilas, A. A., Koutchmy, S., \& Alissandrakis, C. E. 1999, A\&A, 341, 610

Hansteen, V. H., De Pontieu, B., Rouppe van der Voort, L. H. M., van Noort, M., \& Carlsson, M. 2006, ApJ, 647, L73

Heggland, L., De Pontieu, B., \& Hansteen, V. H. 2007, ApJ, 666, 1267

Hollweg, J. V. 1982, ApJ, 257, 345

Lamb, H., 1909, Proc. London Math. Soc., 7, 122

Lee, D., \& Deane, A. E. 2009, J. Comput. Phys., 228, 952

Martinez-Sykora, J., Hansteen, V., De Pontieu, B., \& Carlsson, M. 2009, ApJ, 701,1569

Murawski, K., 2002, Analytical and numerical methods for wave propagation in fluids (Singapore: World Scientific)

Murawski, K., \& Zaqarashvili, T. V. 2010, A\&A, 519, A8

Pasachoff, J. M., Jacobson, W. A., \& Sterling, A. C. 2009, Sol. Phys., 260, 59

Pike, C. D., \& Harrison, R. A. 1997, Sol. Phys., 175, 457

Rouppe van der Voort, L. H. M., Leenaarts, J., De Pontieu, B., Carlsson, M., \& Vissers, G. 2009, ApJ, 705, 272

Shibata, K., \& Suematsu, Y. 1982, A\&A, 78, 333

Sterling, A. C. 2000, Sol. Phys., 196, 79

Suematsu, Y. 1998, Solar Jets and Coronal Plumes, ESA SP, 421, 19

Suematsu, Y., Wang, H., \& Zirin, H. 1995, ApJ, 450, 411

Suematsu, Y., Ichimoto, K., Katsukawa, Y., et al. 2008, ASP Conf. Ser., 397, 27

Tanaka, K. 1974, in Chromospheric Fine Structure, ed. G. Athay, IAU Symp., 56,239

Toro, E. 2009, Riemann solvers and numerical methods for fluid dynamics (Springer)

Tsiropoula, G., Alissandrakis, C. E., \& Schmieder, B. 1994, A\&A, 290, 294

Tziotziou, K., Tsiropoula, G., \& Mein, P. 2003, A\&A, 402, 361

Tziotziou, K., Tsiropoula, G., \& Mein, P. 2004, A\&A, 423, 1133

Vernazza, J. E., Avrett, E. H., \& Loeser, R. 1981, ApJ, 45, 635

Wilhelm, K. 2000, A\&A, 360, 351

Zaqarashvili, T. V., \& Erdélyi, R. 2009, Space Sci. Rev., 149, 355 